\title{
Supercurrent-induced temperature gradient across a nonequilibrium SNS Josephson junction
}

\author{
M.S. Crosser,${ }^{1}$ Pauli Virtanen, ${ }^{2}$ Tero T. Heikkilä, ${ }^{2}$ and Norman O. Birge ${ }^{1, \text { 凡 }}$ \\ ${ }^{1}$ Department of Physics and Astronomy, Michigan State University, East Lansing, MI 48824-2320, USA \\ ${ }^{2}$ Low Temperature Laboratory, Helsinki University of Technology, P. O. Box 2200, FIN-02015 TKK, Finland
}

(Dated: January 13, 2019)

\begin{abstract}
Using tunneling spectroscopy, we have measured the local electron energy distribution function in the normal part of a superconductor-normal metal-superconductor (SNS) Josephson junction containing an extra lead to a normal reservoir. In the presence of simultaneous supercurrent and injected quasiparticle current, the distribution function exhibits a sharp feature at very low energy. The feature is odd in energy, and odd under reversal of either the supercurrent or the quasiparticle current direction. The feature represents an effective temperature gradient across the SNS Josephson junction that is controllable by the supercurrent.
\end{abstract}

PACS numbers: 74.50.+r, 73.23.-b, 85.25.Am, 85.25.Cp

The study of nonequilibrium phenomena in superconductors has a rich history extending over several decades 1]. Advances in microfabrication techniques in the 1990's have broadened the range of possible experiments. In 1999, Baselmans et al. 2] turned a superconductingnormal-superconducting Josephson junction (SNS JJ) into a " $\pi$-junction" by driving the electron energy distribution far from equilibrium with a normal control current applied perpendicular to the supercurrent in a fourterminal geometry. Later, Huang et al. [3] produced similar behavior in a three-terminal sample with the control current injected from a single normal reservoir. The physical explanation behind the $\pi$-junction [4] $[5]$ is the same in the two geometries: the electron energy distribution in the presence of the control current has a doublestep shape [6], which selectively populates the Andreev bound states that carry current in the opposite direction to that in equilibrium.

While the physics of the three-terminal and fourterminal $\pi$-junctions is similar, the current flow and energy distributions are different in the two geometries. In the four-terminal experiment, the control current and supercurrent coexist only in the region where the two wires cross. In the three-terminal experiment, the wire joining the two superconductors carries both supercurrent and normal (quasiparticle) current 7]. It was predicted in Ref. 8 that this coexistence of quasiparticle current and supercurrent will lead to a left-right asymmetry in the effective temperature, due to mixing of the even and odd components of the distribution function, $f(E)$. In this process, supercurrent redistributes the Joule heat due to the quasiparticle current between the different parts of the system. As a result, the double-step distribution function is distorted, and in the absence of inelastic scattering, this distortion is approximately proportional to the energy spectrum of the supercurrent-carrying states. We present here the first measurement of the distortion in $f(E)$, using a local tunnel probe.

Fig. 1a shows the three-terminal sample geometry. A

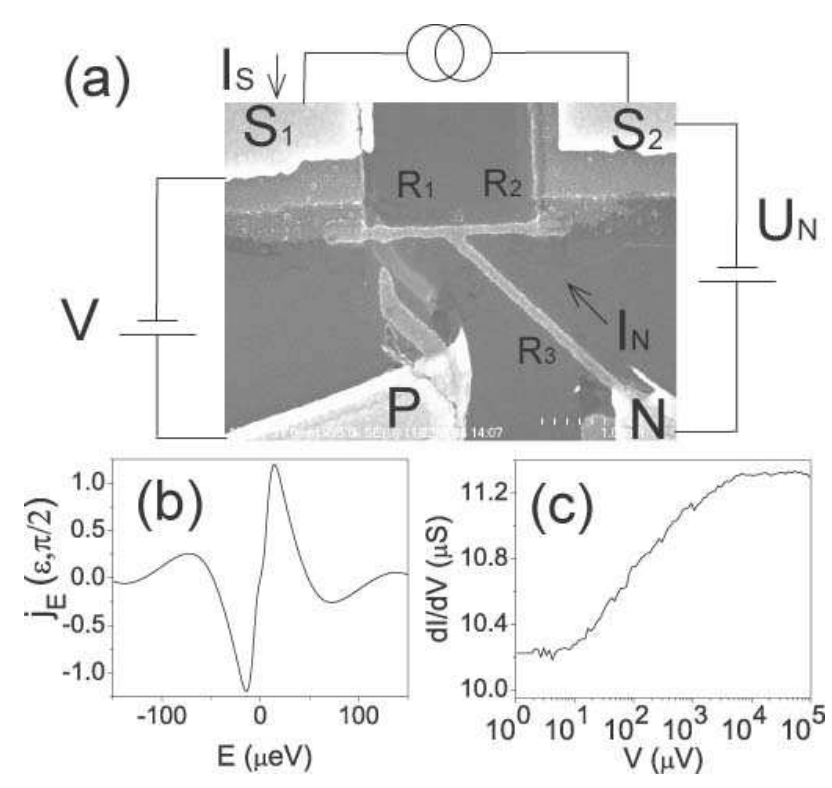

FIG. 1: (a) SEM micrograph of a sample and schematic measurement circuit diagram. (b) Spectral supercurrent $j_{E}$ for $\phi=\pi / 2$ calculated numerically for the sample shown in (a). (c) $\mathrm{dI} / \mathrm{dV}$ of the tunnel probe $(\mathrm{P})$ measured at $\mathrm{B}=0.4 \mathrm{~T}$, where superconductivity is suppressed. The $10 \%$ reduction in $\mathrm{dI} / \mathrm{dV}$ at low bias is due to dynamical Coulomb blockade, as discussed in the text.

normal metal wire connects two superconducting electrodes $\left(S_{1}\right.$ and $\left.S_{2}\right)$ and a normal reservoir $(N)$. The superconducting electrodes are kept at the same chemical potential, which we define as the zero of energy. With the normal electrode biased at a potential $U_{N}$, the distribution function $f(E)$ can be calculated easily in the absence of supercurrent, energy relaxation, and proximity effects. In the normal reservoir, $f(E)=f_{F D}\left(E+e U_{N}\right)$ is a FermiDirac function displaced in energy by $-e U_{N}$. Define the even and odd (in energy) components of $f(E)$ as follows: $f_{T}(E) \equiv 1-f(E)-f(-E)$ and $f_{L} \equiv f(-E)-f(E)$. 
The boundary conditions at the NS interfaces for energies below the superconducting gap, $\Delta$, are $f_{T}=0$ and $\partial f_{L} / \partial x=0$, assuming high-transparency interfaces, no charge imbalance in the superconductors, and no heat transport into the superconductors [9]. The solution for $f(E)$ at the NS interface is the double-step shape: $f(E)=0.5 *\left[f_{F D}\left(E+e U_{N}\right)+f_{F D}\left(E-e U_{N}\right)\right]$.

In the presence of proximity effects and supercurrent, the kinetic equations become more complicated [10]:

$$
\begin{aligned}
& \frac{\partial j_{T}}{\partial x}=0, j_{T} \equiv D_{T}(x) \frac{\partial f_{T}}{\partial x}+j_{E} f_{L}+T(x) \frac{\partial f_{L}}{\partial x} \\
& \frac{\partial j_{L}}{\partial x}=0, \quad j_{L} \equiv D_{L}(x) \frac{\partial f_{L}}{\partial x}+j_{E} f_{T}-T(x) \frac{\partial f_{T}}{\partial x}
\end{aligned}
$$

The spectral charge and energy currents, $j_{T}(E)$ and $j_{L}(E)$, are conserved only in the absence of energy relaxation. The energy-dependent coefficients $D_{T}, D_{L}, j_{F}$, and $T$ can be calculated from the Usadel equation [11], and vary with the superconducting phase difference $\phi$ between $S_{1}$ and $S_{2}$. Fig. 1b shows the spectral supercurrent density, $j_{E}$, for $\phi=\pi / 2$ obtained from a numerical solution of the Usadel equation appropriate for our sample. In the presence of the proximity effect, the kinetic equations must be solved numerically. But one can understand the origin of the effective temperature gradient predicted in Ref. 8 by noting that the terms with $j_{E}$ mix $f_{T}$ and $f_{L}$ in the kinetic equations. If we neglect the $T$ terms and ignore the energy dependence in $D_{T}$ and $D_{L}$, then Eqs. (1a) and (1b) are easily solved analytically. The solution shows that $f_{L}$ along the horizontal wire connecting the two superconductors contains a spatially antisymmetric contribution proportional to $j_{E}$.

The experiments described in this paper were performed on four samples, with similar results obtained on all four. Most of the data reported here were taken from the sample shown in Fig. 1a, with $R_{1}=R_{2}=7.0 \Omega$, $R_{3}=16.9 \Omega$ and tunnel resistance $R_{T}=115 k \Omega$. The samples were fabricated using a PMMA stencil patterned by electron-beam lithography and multiple-angle evaporations [12. The three-legged normal $\mathrm{Ag}$ wire is $70 \mathrm{~nm}$ wide and $30 \mathrm{~nm}$ thick. The superconducting Al electrodes are $60 \mathrm{~nm}$ thick, separated by a distance of $1.4 \mu \mathrm{m}$. The normal reservoir is $200 \mathrm{~nm}$ thick. The tunnel probe (connected to the pad labelled P) is $20 \mathrm{~nm}$-thick Al oxidized in 80 mbar of $\operatorname{Ar}(90 \%)-\mathrm{O}_{2}(10 \%)$ for 6 minutes before deposition of the $\mathrm{Ag}$ wire.

We use tunneling spectroscopy to measure the shape of the distribution function of the $\mathrm{Ag}$ wire at a position close to the superconducting reservoir $S_{1}$, in a manner similar to that in Ref. 6. A complication in our experiment is that the density of states in the Ag wire is strongly modified by proximity to $S_{1}$ and $S_{2}$. The current-voltage characteristic of the probe tunnel junction is

$$
\begin{aligned}
I(V)= & -\frac{1}{e R_{T}} \int d E n_{S}(E) \int d \varepsilon P(\varepsilon) \\
& {\left[f_{S}(E) n_{N}(E-e V-\varepsilon)\left(1-f_{N}(E-e V-\varepsilon)\right)\right.} \\
- & \left.\left(1-f_{S}(E)\right) n_{N}(E-e V+\varepsilon) f_{N}(E-e V+\varepsilon)\right]
\end{aligned}
$$

where $R_{T}$ is the normal state tunnel resistance, $n_{N}$ and $n_{S}$ are the normalized densities of states, and $f_{N}$ and $f_{S}$ are the electron energy distribution functions on the $\mathrm{Ag}$ and $\mathrm{Al}$ sides of the tunnel junction, respectively. Eq. (2) includes the effects of "dynamical Coulomb blockade" 13 through the quantity $P(\varepsilon)$, the probability for an electron to lose energy $\varepsilon$ to the resistive environment while tunneling through the barrier.

Our strategy was to perform a series of experiments in which all of the quantities in Eq. (2) are known except for one, and then deconvolve the data to find the missing quantity. The first step is to find $P(\varepsilon)$, which can be determined from $d I / d V(V)$ data with a large magnetic field $B$ to suppress superconductivity. Fig. 1c shows $d I / d V$ vs. $V$ with $B=0.4 \mathrm{~T}$. In agreement with previous work [14], $d I / d V$ depends logarithmically on $V$ over nearly three decades of voltage. For a tunnel junction in a resistive environment $R$, with $R \ll R_{K}=h / e^{2}$, the function $P(\varepsilon)$ is well approximated by:

$$
P(\varepsilon)=\left\{\begin{array}{cl}
\frac{\alpha}{E_{0}}\left(\frac{\varepsilon}{E_{0}}\right)^{\alpha-1} & , \varepsilon<E_{0} \\
0 & , \varepsilon>E_{0}
\end{array}\right.
$$

where $\alpha=2 R / R_{K}$ and $E_{0}=e^{2} /(\pi \alpha C)$. The $\mathrm{dI} / \mathrm{dV}$ data in Fig. 1c can be fit to this form for $P(\varepsilon)$ with $\alpha=0.0176$ and $E_{0}=2.0 \mathrm{meV}$, which correspond to the reasonable values $R=230 \Omega$ and $C=1.4 \mathrm{fF}$.

The second step is to determine $n_{N}(E)$, the density of states of the $\mathrm{Ag}$ wire in close proximity to $S_{1}$. In equilibrium, $f_{S}(E)$ and $f_{N}(E)$ are Fermi-Dirac functions at a temperature close to that of the cryostat $(35 \mathrm{mK})$. In most of our samples, the $d I / d V(V)$ data in zero applied magnetic field show an anomalous sharp feature at $e V \approx \Delta$, which disappears when $B>10 \mathrm{mT}[15]$; hence, all the low-field data reported in this paper were taken with $B=12.5 \mathrm{mT}$. We use the standard BCS form for $n_{S}(E)$ of the $\mathrm{Al}$ probe, with a small depairing parameter to account for the applied magnetic field [16]. To determine $n_{N}(E)$ without ambiguity, we must know the gap $\Delta$ of the Al probe. For that purpose we fabricated, on the same chip, a reference SIN tunnel junction with no proximity effect on the $\mathrm{N}$ side. The $d I / d V$ data for the SIN junction, shown in the right inset of Fig. 2] could be fit perfectly with Eq. (2) assuming a BCS form for $n_{S}(E)$ with $\Delta=274 \mu \mathrm{eV}$, a depairing parameter of $\gamma \equiv \Gamma / \Delta=0.0020$ and with Dynamical Coulomb Blockade parameters determined from high-field measurements on that junction. The rather large value of $\Delta$ is common for thin thermally-evaporated $\mathrm{Al}$ films [17]. $d I / d V$ data 
for the sample tunnel junction, shown in the left inset to Fig. 2, looks noticeably different from the $d I / d V$ data on the reference SIN tunnel junction due to proximity effect in the $\mathrm{Ag}$ wire. With $\Delta$ determined, the $d I / d V$ data can be deconvolved to produce $n_{N}(E)$ of the $\mathrm{Ag}$ wire, shown in the main panel of Fig. 2. Equilibrium $d I / d V$ measurements were repeated with supercurrent passing through the SNS JJ, since $n_{N}(E)$ depends on $\phi$, the phase difference between $S_{1}$ and $S_{2}$. Fig. 2 also shows $n_{N}(E)$ obtained with $I_{S}=0.9 I_{c}$ and $-0.9 I_{c}$, which are indistinguishable from each other.

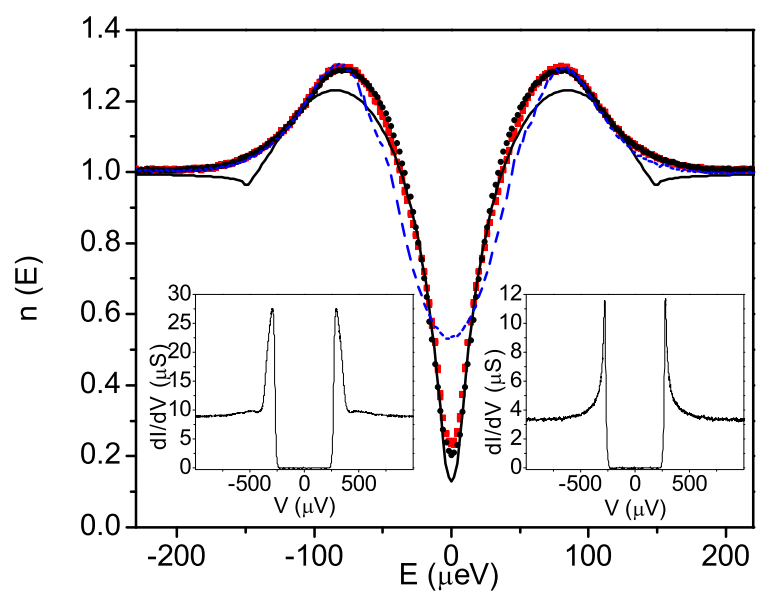

FIG. 2: (Color online) The $n_{N}(E)$ of the $\mathrm{Ag}$ wire at the position of the probe electrode, with (red) and without (black) supercurrent passing through the SNS Josephson junction, obtained from $\mathrm{dI} / \mathrm{dV}$ data such as shown in the left inset. The solid line is a fit to the solution of the Usadel equation discussed in the text. The (blue) dashed line shows $n_{N}(E)$ needed to fit nonequilibrium data with $U_{N}=53 \mu \mathrm{V}$. Right inset: $\mathrm{dI} / \mathrm{dV}$ data on a reference SIN tunnel junction, used to determine the gap $\Delta$ of the superconducting probe.

To obtain a decent fit of the data in Fig. 2 to the density of states calculated from the Usadel theory, we need to assume a considerably smaller value of $\Delta \approx 150 \mu \mathrm{eV}$ in the superconducting reservoirs feeding the supercurrent than that measured with the SIN reference junction. The reason for this smaller value is the presence of a normal-metal-superconductor bilayer close to these reservoirs (see Fig. (1). Once we know this $\Delta$, we find the Thouless energy $E_{T}=\hbar D / L_{S}^{2}$ characterizing the normal-metal wire of length $L_{S}$ between the superconductors by fitting the temperature dependent supercurrent at $U_{N}=0$ analogously to Ref. 3 . The quality of the fit is fairly insensitive to the precise value of $\Delta$, and we obtain $E_{T}=5.56 \mu \mathrm{eV}$. In the fit, we also corrected for the finite size of the superconducting terminals and calculated the position-dependent order parameter $\Delta$ selfconsistently close to the NS interface. Once we know these two energy scales, we can calculate the local density of states $n_{N}(E)$ at the position of the probe. As there is some ambiguity about the position due to the finite normal-metal-superconductor overlap, we fit also this within the limits set by the sample geometry. This fit is shown as a solid line in Fig. 2.

The third step in the experiment is to apply a voltage $U_{N}$ to the normal reservoir, and repeat the $\mathrm{dI} / \mathrm{dV}$ measurements across the probe junction with various values of $U_{N}$ and $I_{S}$. Fig. [3 shows three sets of $d I / d V$ vs. $V$ data with $U_{N}=53 \mu \mathrm{V}$, and with $I_{S}=0,-0.9 I_{c}$, and $+0.9 I_{c}$. The double peaks in $d I / d V$ near $V= \pm \Delta$ arise from the double-step shape in $f_{N}(E)$, the distribution function in the $\mathrm{Ag}$ wire. Since we know $P(\varepsilon), n_{S}(E)$, and $n_{N}(E)$, and since $f_{S}(E)$ is a Fermi-Dirac function, we planned to determine $f_{N}(E)$ from a deconvolution of these $d I / d V$ data. Instead, we found that we were unable to fit the data shown in Fig. 3 using Eq. (2), with any physically permissible form for $f_{N}(E)$. Only by allowing $n_{N}(E)$ to vary slightly from the form obtained in equilibrium (dashed line in Fig. 2), could we fit the nonequilibrium data.

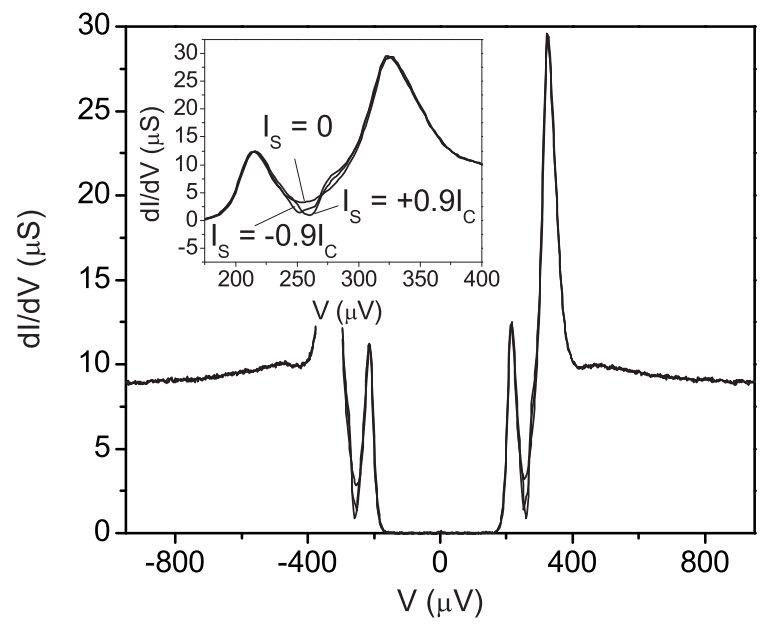

FIG. 3: dI/dV data of the probe tunnel junction with the normal reservoir biased at $U_{N}=53 \mu \mathrm{V}$. Inset: Enlarged view of the double-peak at positive bias, showing the differences between the data sets taken with $I_{S}=0$ and $\pm 0.9 I_{c}$.

Fortunately, we can extract the supercurrentdependent changes in $f_{N}(E)$ by considering differences between data sets with opposite values of $I_{S}$. The equilibrium measurements shown in Fig. 2 confirm our expectation that $n_{N}\left(E, U_{N}=0, I_{S}\right)=n_{N}\left(E, U_{N}=\right.$ $\left.0,-I_{S}\right)$. This symmetry holds approximately also for $U_{N} \neq 0$; hence the quantity $d I / d V\left(V, U_{N}, I_{S}\right)-$ $d I / d V\left(V, U_{N},-I_{S}\right)$ depends only on the difference $\delta f_{N}(E) \equiv f_{N}\left(E, U_{N}, I_{S}\right)-f_{N}\left(E, U_{N},-I_{S}\right)=\delta f_{L}(E)$, the change in the function $f_{L}(E)$. The left panel of Fig. 4 shows $\delta f_{N}$ defined in this way, with $U_{N}=22 \mu \mathrm{V}$ and $I_{S}=0.9 I_{c}$. There are sharp peaks at $E \approx \pm 15 \mu \mathrm{V}$, odd in energy. This feature in $f_{N}(E)$ represents changes 
in the local effective electron temperature, according to Eq. (5) in Ref. 8]. To confirm that the peaks are not due to a geometrical asymmetry in the sample, such as a flaw in the fabrication process, a second determination of $\delta f_{N}(E)$ is shown superposed on the first. This second form was obtained by reversing the signs of both $U_{N}$ and $I_{S}: f_{N}\left(E,-U_{N},-I_{S}\right)-f_{N}\left(E,-U_{N}, I_{S}\right)$. The two data sets are in near perfect agreement.
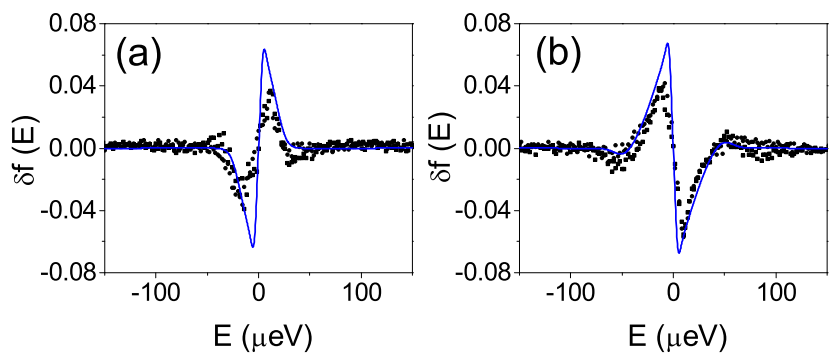

FIG. 4: (a) $\delta f(E) \equiv f_{N}\left(E, U_{N}, I_{S}\right)-f_{N}\left(E, U_{N},-I_{S}\right)$ for $U_{N}=22 \mu \mathrm{V}$ and $I_{S}=0.9 I_{c}$. (b) Same quantity for $U_{N}=$ $53 \mu \mathrm{V}$ and $I_{S}=0.9 I_{c}$, with the SNS JJ in the $\pi$ state. Notice the sign change between the two data sets. In both figures, a second data set is shown with the signs of both $U_{N}$ and $I_{S}$ reversed. Solid lines are numerical solutions to Eq. (1).

If we increase $U_{N}$, we can convert the SNS JJ to a $\pi$ junction [3]. The SNS JJ crosses over from the 0-state to the $\pi$-state at $U_{N}=30 \mu \mathrm{V}$. The right panel of Fig. 4 shows $\delta f_{N}(E)$ data in the $\pi$-state for $U_{N}=53 \mu \mathrm{V}$ and $I_{S}=0.9 I_{c}$. Compared to Fig. 4a, the sign of the lowenergy feature in $\delta f_{N}(E)$ is reversed, demonstrating that the phase difference $\phi$, rather than the supercurrent, determines the sign of the effective temperature gradient across the SNS JJ.

Let us compare these results to those obtained from Eqs. (1) using the parameters $E_{T}, \Delta$ obtained from the previous fits, with no additional fitting. The computed theory curves are shown as solid lines in Fig. 4 and they agree with the experimental data rather well. Note that for this calculation, we entirely neglected the effect of inelastic scattering.

As stated above, we have recovered $\delta f_{N}(E)$ without knowing the exact form of $n_{N}(E)$. We have verified that our results are rather insensitive to small changes in $n_{N}(E)$ by performing the deconvolution using several different forms for $n_{N}(E)$, with the value of $n_{N}(0)$ varying from 0.1 to 0.5 . The amplitude of the oscillations in $\delta f_{N}(E)$ are inversely proportional to $n_{N}(E)$ near $E=0$, but the overall shape does not change from those shown in Fig. 4. To extract the full $f_{N}(E)$ from our data would require a separate measurement of $n_{N}(E)$ (using a normal tunnel probe [18]) at each value of $U_{N}$ and $I_{S}$ used in our experiment. As $U_{N}$ increases, deviations of $n_{N}(E)$ from its equilibrium form may arise from nonequilibrium processes in the superconducting reservoirs, where quasi- particle current is converted to supercurrent [19].

The feature we have measured has been called a "Peltier-like" effect in Ref. 8, because an applied current induces a temperature gradient. It should be emphasized, however, that our experiment is far from equilibrium. We are not measuring a linear response transport coefficient, as was done with the thermopower of Andreev interferometers [20, 21]. It is unlikely that one could achieve real local cooling in our experiment, as one does with the conventional Peltier effect. Perhaps the most intriguing aspect of our results is that the nonequilibrium feature in $f(E)$ shown in Fig. 4 provides an approximate visualization of the spectral supercurrent density $j_{E}-$ a quantity whose energy dependence has previously been inferred only indirectly through $\pi$-junction experiments.

We are grateful to F. Wilhelm, F. Pierre, and S. Yip for fruitful discussions. N.B. also thanks H. Pothier and D. Esteve for their long-standing collaboration. This work was supported by NSF grants DMR-0104178 and 0405238, by the Keck Microfabrication Facility supported by NSF DMR-9809688, and by the Academy of Finland.

* Electronic address: birge@pa.msu.edu

[1] K. E. Gray, ed., Nonequilibrium superconductivity, phonons, and Kapitza boundaries (Plenum Press, New York, 1981).

[2] J. J. A. Baselmans et al., Nature 397, 43 (1999).

[3] J. Huang et al., Phys. Rev. B 66, 020507(R) (2002).

[4] S. K. Yip, Phys. Rev. B 58, 5803 (1998).

[5] F. K. Wilhelm, G. Schön, and A. D. Zaikin, Phys. Rev. Lett. 81, 1682 (1998).

[6] H. Pothier et al., Phys. Rev. Lett. 79, 3490 (1997).

[7] R. Shaikhaidarov et al., Phys. Rev. B 62 R14649 (2000).

[8] T. T. Heikkilä, T. Vänskä, and F. K. Wilhelm, Phys. Rev. B 67, 100502(R) (2003).

[9] A. F. Andreev, Sov. Phys. JETP 19(5), 1228 (1964).

[10] P. Virtanen and T. T. Heikkilä, J. Low Temp. Phys. 136, 401 (2004), and references therein.

[11] K. D. Usadel, Phys. Rev. Lett. 25, 507 (1970).

[12] G. J. Dolan and J. H. Dunsmuir, Physica B 152, 7 (1988).

[13] G. L. Ingold and Y. V. Nazarov, Single Charge tunneling Coulomb Blockade phenomena in Nanostructures, vol. 294 (Plenum Press, London, 1992).

[14] A. Anthore et al., Phys. Lett. 90, 076806 (2003).

[15] F. Pierre, Ann. Phys. (Paris) 26, No. 4 (2001), Ch. 3.

[16] A. Anthore, H. Pothier, and D. Esteve, Phys. Rev. Lett. 90, 127001 (2003).

[17] P.M. Tedrow et al., Phys. Rev. Lett. 52, 1637 (1984).

[18] S. Gueron et al., Phys. Rev. Lett. 77, 3025 (1996)

[19] M. Yu and J. Mercereau, Phys. Rev. Lett. 28, 1117 (1972); J. Clarke, Phys. Rev. Lett. 28, 1363 (1972).

[20] D. A. Dikin, S. Jung, and V. Chandrasekhar, Europhys. Lett. 57, 564 (2003); A. Parsons, I.A. Sosnin, and V.T. Petrashov, Phys. Rev. B 67, 140502(R) (2003).

[21] R. Seviour and A.F. Volkov, Phys. Rev. B 62, R6116 (2000); P. Virtanen and T. T. Heikkilä, Phys. Rev. Lett. 92, 177004 (2004). 